ARTículo

\title{
Educación Superior en México: los retos del gobierno presidencial en el periodo 2018-2024 en materia de cobertura
}

\author{
Juan Carlos Castellanos-Ramírez a \\ Shamaly Alhelí Niño Carrasco ${ }^{b}$
}

\section{Resumen}

Una de las prioridades del actual Gobierno de México es incrementar, para el año 2024, la tasa de cobertura en Educación Superior al 100\%. Esta meta implica transformaciones importantes tanto en la organización de las universidades como en el funcionamiento y operación de los programas educativos. En este artículo se presenta un análisis sobre los desafíos que enfrenta el actual gobierno mexicano en materia de cobertura en Educación Superior y la poca evolución que ha tenido durante la última década; a partir de dicho análisis se proponen cinco ejes estratégicos que contribuyen a la construcción de políticas educativas dirigidas al fortalecimiento de la Educación Superior y la ampliación de la matrícula dentro de las universidades. Se sugiere que para incrementar la tasa de cobertura es necesario el desarrollo de políticas dirigidas al fortalecimiento y la diversificación de programas educativos en línea dentro de las universidades.

Palabras clave: Cobertura Educativa. Educación en Línea. Educación Superior. Universidades de México.

\section{Introducción}

Uno de los principales propósitos que el Gobierno de México asumió para el sexenio 2018-2024 es garantizar el acceso a la Educación Superior a todos los jóvenes del país. En este sentido, el actual Presidente de México, Lic. Andrés Manuel López Obrador, ha manifestado en repetidas ocasiones que al finalizar

\footnotetext{
a Universidad Autónoma de Baja California, Mexicali, Baja California, México.

b Universidad Autónoma de Baja California, Mexicali, Baja California, México. 
su sexenio gubernamental se alcanzará una cobertura del 100\% en dicho nivel educativo; sin embargo, se desconocen las estrategias que habrán de implementarse para alcanzar dicha meta.

Si bien es cierto que en esta línea se han realizado algunos pronunciamientos por parte del titular de la Secretaría de Educación Pública, como la construcción de 100 universidades, la eliminación de exámenes de admisión y la implementación de un nuevo programa de becas, hasta el momento no se ha llevado a cabo un análisis profundo sobre la situación actual de las instituciones de Educación Superior en materia de cobertura, de las necesidades económicas, tecnológicas y humanas por las que atraviesan, ni de las posibles vías alternas para mejorar su crecimiento. En este sentido, de acuerdo con Miranda Lopes (2018), cuando los mandatos de ley no son suficientes, se requiere el desarrollo de acciones de política pública para garantizar el acceso a la Educación Superior.

Considerando la premisa anterior, en este trabajo se sugiere, como una estrategia de ampliación de la cobertura educativa, el desarrollo de políticas dirigidas al fortalecimiento y la diversificación de programas educativos en línea. Esta propuesta se sustenta a partir del análisis de la cobertura y la reflexión de los problemas actuales que atraviesan las instituciones de Educación Superior del país, ya que para lograr la equidad y la calidad de la Educación se deben conjuntar "diversos esfuerzos sociales e institucionales que permitan definir una ruta viable en el mediano y largo plazos" (MIRANDA LOPES; ISLAS DOSSETTI, 2016, p. 152).

En el primer apartado se plantea la cobertura como uno de los aspectos clave de las políticas de inclusión educativa. En el segundo apartado se presentan datos generales sobre la cobertura educativa en los distintos niveles educativos y algunos rasgos en torno a la evolución de la cobertura en Educación Superior durante los últimos 14 años. En el tercer apartado se abordan, por un lado, las tendencias internacionales de cobertura considerando la situación de México en contraste con otros países y, por otro lado, las tendencias nacionales contrastando los niveles de cobertura de las entidades federativas de México. En el cuarto apartado se plantean los desafíos que enfrenta el nuevo gobierno para alcanzar la cobertura total en Educación Superior y se destacan cinco ejes estratégicos para la consecución de dicha meta. En el quinto apartado se propone el fortalecimiento de la Educación en línea como una de las políticas que permitirá a las instituciones de Educación Superior brindar mayor cobertura educativa bajo el principio de responsabilidad social. En el último apartado se realizan los comentarios finales del trabajo destacando los aportes más relevantes. 


\section{Políticas de inclusión educativa: cobertura, diversidad y calidad}

En las últimas dos décadas, las políticas de inclusión educativa han ocupado un papel central en las agendas gubernamentales de los países. De acuerdo con las directrices sobre políticas de inclusión emitidas en 2009 por la Organización de las Naciones Unidas para la Educación, la Ciencia y la Cultura (Unesco, por sus siglas en inglés), los gobiernos deben considerar tres líneas de acción para garantizar la inclusión educativa:

- Cobertura: los gobiernos deben crear mecanismos que garanticen a toda la población el acceso al sistema educativo nacional (UNICEF, 2006).

- Diversidad: el sistema educativo nacional debe responder a la diversidad de condiciones y necesidades económicas, culturales, religiosas, cognoscitivas, entre otros aspectos, de los estudiantes (MARCHESI et al., 2014).

- Calidad: se deben crear condiciones para que la cobertura educativa sea de calidad; no basta con tener espacios en las aulas para que asistan los estudiantes, sino que además deben contar con modelos educativos que propicien aprendizajes significativos en los estudiantes (OROZCO CRUZ et al., 2009).

Mientras que los gobiernos de países desarrollados han garantizado a sus ciudadanos los dos primeros niveles de inclusión educativa (cobertura y diversidad) centrando sus políticas actuales al logro de la calidad académica, en el caso de los países subdesarrollados los gobiernos siguen esforzándose por lograr el primer nivel de inclusión correspondiente a la cobertura educativa, principalmente en el nivel superior (RAMOS SERPA; LOPEZ FALCÓN, 2019).

\section{Cobertura educativa en los distintos niveles educativos}

En el caso de México, durante los últimos 15 años la cobertura educativa ha mostrado un mayor crecimiento en lo que respecta al nivel de Educación Básica, en contraste con los niveles de Educación Media Superior y Superior. Para el ciclo 2015-2016, la tasa neta de cobertura en Educación Primaria fue del 98,7\%, mientras que para el nivel secundaria fue del 87,5\% (MÁRQUEZ JIMÉNEZ, 2017).

Por su parte, en los niveles educativos subsiguientes no se ha logrado la cobertura internacional promedio establecida por la Organización para la Cooperación 
y el Desarrollo Económicos (OCDE), la cual se estima por arriba del 65\% en valores de tasa neta y del $80 \%$ en valores de tasa bruta. En México, la tasa neta de cobertura en Educación Media Superior para el ciclo escolar 2015-2016 fue del $59,5 \%$ (SOLÍS, 2018), lo que en frecuencias reales significa que una población de 2,720,656 jóvenes de entre 15 a 17 años no tiene acceso a la Educación Media Superior (INEE, 2016).

Por otra parte, en la Educación Superior el panorama es aún más desalentador. De acuerdo con cifras de la Secretaría de Educación Pública, para el ciclo escolar 2017-2018 se estimó una cobertura bruta del 38,4\% (GONZÁLEZ, 2018; MEXICO, 2017). Como puede observarse en la Figura 1, del año 2005 a la fecha, la cobertura en Educación Superior ha permanecido prácticamente estancada, ya que el crecimiento anual representa el uno por ciento aproximadamente.

Figura 1 - Crecimiento de la cobertura de la Educación Superior en México del año 2005 al 2018

Cobertura en educación superior

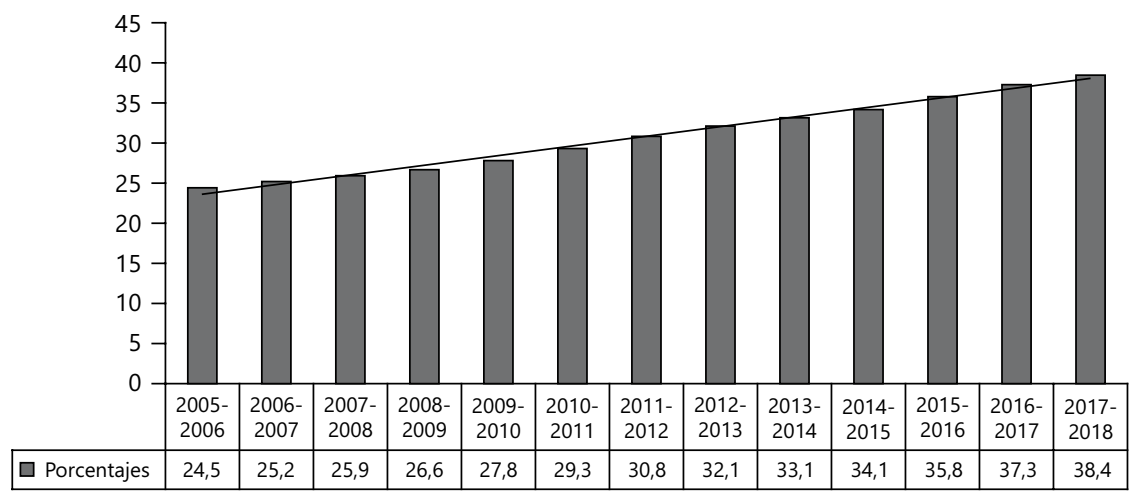

Fuente: Elaboración propia, a partir de los datos de Anuies (2018a) y Mendoza Rojas (2018).

De acuerdo con los datos aportados por la Asociación Nacional de Universidades e Instituciones de Educación Superior (Anuies), México tiene un atraso en materia de cobertura de Educación Superior (ANUIES, 2018b) y, hasta el día de hoy, no ha sido posible alcanzar el porcentaje promedio de cobertura bruta, equivalente al $48 \%$, que sostienen los países latinoamericanos y del Caribe.

En los inicios de la administración gubernamental del expresidente Enrique Peña Nieto (2012-2018), se estableció como meta que para el año 2018 se 
alcanzaría, al menos, el 45\% de cobertura en Educación Superior (GONZÁLEZ, 2015); sin embargo, al término de su administración dicha cobertura solo representó el 38,4\%. Con estas cifras, México está lejos de igualar las tasas actuales de Argentina (85,7\%), Chile (90,3\%), Costa Rica (54,0\%), Uruguay $(55,6 \%)$, Colombia $(58,7 \%)$, Ecuador $(41,0 \%)$, entre otros (SÁNCHEZ JIMÉNEZ, 2018).

\section{Tendencias internacionales y nacionales de cobertura en la Educación Superior}

De acuerdo con el informe "Panorama de la Educación 2017" publicado por la OCDE (2017), en los países miembros de dicha organización la media de la población entre 25 a 34 años que posee un título o grado de Educación Superior es equivalente al $44 \%$. Según los datos de la Tabla 1 , México se sitúa muy por debajo de los porcentajes observados en el resto de los países miembros de la OCDE, ya que tan solo el $24 \%$ de la población mexicana cuenta con un título o grado de Educación Superior.

Tabla 1 - Población entre 25 a 34 años que cuenta con un título o grado de Educación Superior según el estudio realizado por la OCDE en el año 2016

\begin{tabular}{lccccc}
\hline Igual o superior a la media* & \multicolumn{2}{c}{ Inferior a la media* } & \multicolumn{2}{c}{ Inferior al 25\% } \\
\hline Corea & $70 \%$ & Estonia & $42 \%$ & México & $24 \%$ \\
Canadá & $62 \%$ & Grecia & $42 \%$ & Argentina & $19 \%$ \\
Rusia & $60 \%$ & España & $42 \%$ & China & $18 \%$ \\
Lituania & $55 \%$ & Finlandia & $42 \%$ & Brasil & $18 \%$ \\
Reino Unido & $52 \%$ & Austria & $40 \%$ & India & $14 \%$ \\
Luxemburgo & $52 \%$ & Portugal & $35 \%$ & Indonesia & $13 \%$ \\
Irlanda & $52 \%$ & República & $34 \%$ & Sudáfrica & $11 \%$ \\
Suiza & $49 \%$ & Checa & & & \\
Australia & $49 \%$ & Alemaquia & $34 \%$ & & \\
Israel & $48 \%$ & Hungría & $31 \%$ & & \\
Estados Unidos & $48 \%$ & Turquía & $30 \%$ & & \\
Suecia & $48 \%$ & Chile & $30 \%$ & & \\
Dinamarca & $46 \%$ & Colombia & $29 \%$ & & \\
Francia & $45 \%$ & Costa Rica & $29 \%$ & & \\
Países bajos & $45 \%$ & Italia & $25 \%$ & & \\
& & & &
\end{tabular}


Continuación

\begin{tabular}{lclc}
\hline \multicolumn{2}{l}{ Igual o superior a la media* } & Inferior a la media* & Inferior al 25\% \\
\hline Nueva Zelanda & $45 \%$ & Arabia & $25 \%$ \\
Bélgica & $45 \%$ & & \\
Polonia & $44 \%$ & & \\
Eslovenia & $44 \%$ & & \\
Letonia & $44 \%$ & & \\
Noruega & $44 \%$ & & \\
Islandia & $44 \%$ & & \\
\hline
\end{tabular}

Nota. Según la OCDE, la media de la población de 25 a 34 años que cuenta con un título o grado de Educación Superior equivale al $44 \%$

Fuente: a partir de los datos del OECD (2017)

Aunado a lo anterior, los indicadores internacionales que se muestran en el informe "Visión y acción 2030; propuesta de la Anuies para renovar la Educación Superior en México" (ANUIES, 2018b) con respecto a los niveles de cobertura de Educación Superior tampoco son alentadores, como el propio informe señala:

México tiene un bajo nivel de cobertura de Educación Superior que no favorece el desarrollo y el bienestar de la población. Actualmente nuestro país tiene una cobertura de $38.4 \%$, diez puntos porcentuales debajo de la media de América Latina y el Caribe (48,4\%) [mientras que] Otros países de la región han superado el 50\% (p. 28).

En efecto, según los indicadores internacionales sobre la tasa bruta de cobertura (TBC) de Educación Superior - número de alumnos en edad idónea inscritos en este nivel educativo- que se presentan en la Figura 2, México no solo se sitúa por debajo del promedio mundial (barra correspondiente a la media de la OCDE, con un valor equivalente al $72,8 \%$ ), sino que también se encuentra diez puntos porcentuales por debajo de la TBC de Latinoamérica y el Caribe (48,4\%). Así, con un porcentaje equivalente al 38,4\%, el nivel de cobertura de Educación Superior en México se aleja de países como Chile (90,3\%), Argentina (85,7\%), Colombia $(58,7 \%)$, Uruguay $(55,6 \%)$, Costa Rica $(54,0 \%)$ y Ecuador $(45,5 \%)$. Es importante destacar que las comparaciones internacionales deben hacerse con prudencia y tomar en cuenta no solo el nivel de desarrollo de los países, sino también el tamaño de la población. 
Figura 2 - Tasa bruta de cobertura de Educación Superior en el año 2016

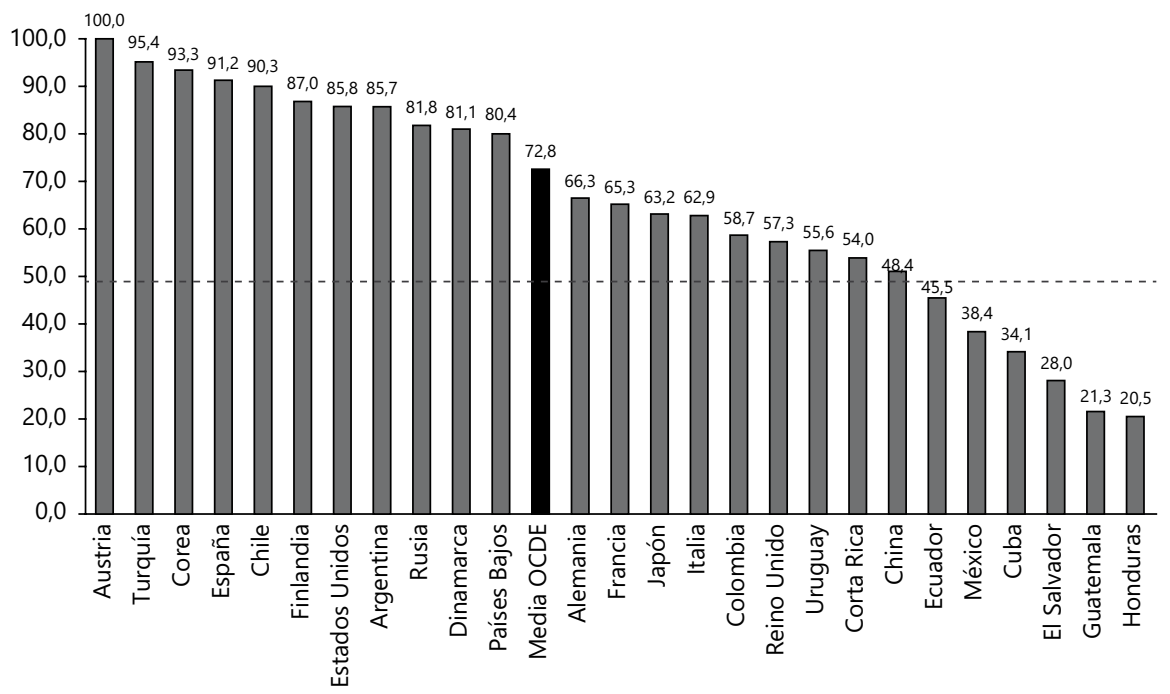

. - . - Tasa bruta de cobertura de Latinoamérica y el Caribe: $48.4 \%$

Fuente: Adaptado de Anuies (2018b, p. 56)

De acuerdo con la Figura 3, nivel nacional también se observan diferencias importantes en el indicador de cobertura de Educación Superior entre entidades federativas, ya que existen grandes brechas entre el número de alumnos en edad idónea inscritos en Educación Superior entre los estados del sur y el resto de México. De hecho, de acuerdo con la Figura 2, en el ciclo 20172018 hay 18 entidades federativas con una TBC inferior al 38,4\% nacional, siendo los más afectados los estados de Oaxaca (19,7\%), Guerrero (21,5\%) y Chiapas $(21,8 \%)$. Por su parte, los estados que presentan porcentajes con una mayor cobertura son Nuevo León $(48,4 \%)$, Sinaloa $(53,0 \%)$ y la Ciudad de México (97,5\%). 
Figura 3 - Tasa bruta de cobertura de Educación Superior a nivel nacional en el ciclo 2017-2018

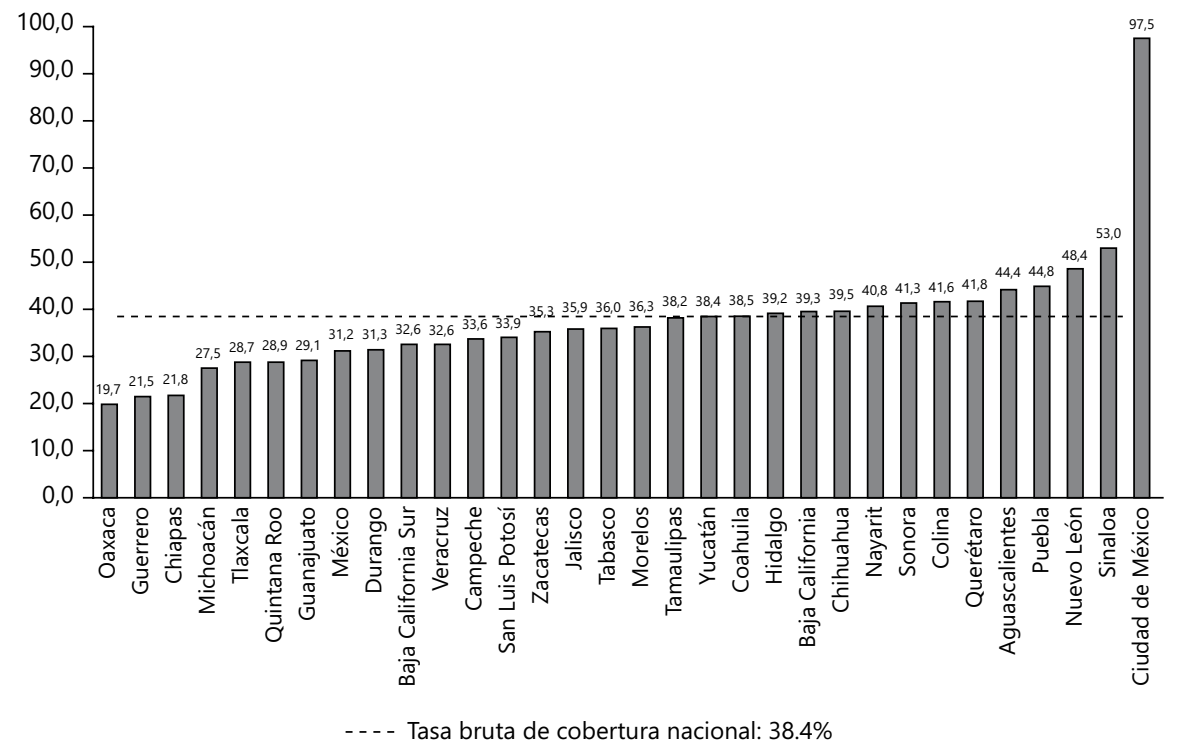

Fuente: Adaptado de Anuies (2018b, p. 64).

\section{Promesas de campaña y desafíos para un nuevo sexenio presidencial en México}

Como se ha señalado en el apartado anterior, el porcentaje de jóvenes que ingresan a la Educación Superior en México es inferior al que se precisa para los países que buscan avanzar hacia el desarrollo social y económico. Como resultado, ante la falta de oportunidades que tienen los jóvenes aspirantes para acceder a la Educación Superior y optar por mejores condiciones laborales, se ha producido una desaceleración en el crecimiento económico del país.

A diferencia de la sociedad industrial en donde la principal fuente de crecimiento económico de los países fue la productividad de las fábricas y la mano de obra de sus trabajadores, en la sociedad de la información el desarrollo económico se basa en el conocimiento y las habilidades del capital humano para producirlo. En efecto, como Moreno-Brid y Ruiz-Nápoles (2010) señalan, la mejora del desarrollo científico, tecnológico y la productividad laboral de un país está fuertemente relacionada con el crecimiento potencial de su economía, por lo que sus políticas deben encaminarse a mejorar los capitales físico y humano; 
es precisamente en este contexto donde la cobertura de la Educación Superior adquiere una relevancia fundamental para el desarrollo de los países.

Retomando la premisa anterior, el gobierno presidencial del Lic. Andrés Manuel López Obrador ha proyectado que durante su sexenio se alcance la cobertura total de la población juvenil con dicho grado escolar. Es así como bajo el lema "Educación para todos" se estableció que durante el actual gobierno el porcentaje de jóvenes rechazados de las universidades disminuya radicalmente, por lo que una de las primeras acciones que se pretende implementar es cancelar los exámenes de ingreso al nivel educativo superior.

Como parte también de las acciones encaminadas a mejorar los porcentajes de cobertura de Educación Superior, bajo el lema "Becarios sí, sicarios no", se busca implementar el nuevo programa de becas "Jóvenes construyendo el futuro"; este programa contempla dos modalidades: de capacitación y educativa. En detalle, la modalidad educativa será coordinada por la Secretaría de Educación Pública (SEP) y se centrará en jóvenes que hayan concluido el bachillerato y busquen acceder a la Educación Superior, a quienes se les dará un apoyo económico mensual. Sin embargo, hasta el momento no se han concretado estrategias claras para la operación e implementación de la modalidad educativa de dicho programa de becas.

Con base en lo anterior, más allá del otorgamiento a corto plazo de becas económicas a jóvenes universitarios, se considera de suma importancia el explorar a fondo las circunstancias que han dificultado la ampliación de la cobertura en Educación Superior en México, de tal manera que las propuestas y acciones resultantes de dicha exploración generen acuerdos conjuntos entre, por una parte, el gobierno federal y gobiernos estatales y, por otra parte, las propias instituciones educativas que ofertan programas educativos de Educación Superior, así como los organismos directamente relacionados con la promoción y el desarrollo de la Educación Superior en México, por ejemplo la Anuies o el Consejo Nacional de Ciencia y Tecnología (Conacyt).

A continuación, se destacan cinco ejes estratégicos que, desde el punto de vista de los autores, contribuirán al fortalecimiento de la Educación Superior y la ampliación de la matrícula dentro de las universidades.

a) Respeto a la autonomía y consenso entre universidades: En primer lugar, debe tomarse en cuenta que el establecimiento de mecanismos de admisión de las universidades públicas, más que una política educativa, ha sido una estrategia implementada por las universidades desde hace unos 30 años ante la 
gran demanda de acceso al nivel superior por parte de los jóvenes aspirantes y la falta de espacios o infraestructura suficiente, así como del personal académico y administrativo para atenderlos (GÓMEZ, 2018). Por lo tanto, antes de aplicar una política verticalista, el gobierno federal debe apostar por el diálogo con las universidades respetando su autonomía, su historia, sus orientaciones académicas $\mathrm{o}$, incluso, los diferentes regímenes legales y fines mediante los cuales fueron fundadas, de tal manera que se siga coadyuvando al cumplimiento de su compromiso social.

Si bien es cierto que la autonomía de las universidades debe servir para promover la obligatoriedad y la gratuidad de los estudios de nivel superior, es una responsabilidad imperativa que el gobierno federal sea coherente, ya que la gratuidad y la obligatoriedad implican un compromiso de financiamiento suficiente

para mantener las actividades de docencia, investigación y extensión en su mejor nivel y alcance. Al mismo tiempo, debe asumir su responsabilidad de aportar los recursos necesarios para ampliar las oportunidades educativas hasta alcanzar la universalización (ORDORIKA SACRISTÁN, 2019, p. 7).

b) Mejora del déficit en la infraestructura y capital humano en las universidades públicas: Ante la crisis económica que enfrentan las familias mexicanas, la gran mayoría de los jóvenes se inclina por opciones de Educación Pública; sin embargo, la demanda de los aspirantes que desean ingresar a la Educación Superior rebasa por mucho las condiciones estructurales -o incluso tecnológicas- y de capital humano de las universidades públicas. Como se ha referido en el punto anterior, estas deficiencias obligan a que cada año se restrinja el número de espacios disponibles para el ingreso de los jóvenes; por mencionar solo un ejemplo, en el ciclo escolar 2016-2017 siete de cada diez estudiantes se quedaron sin un espacio dentro de las universidades, lo que representa -en términos brutos- un rechazo aproximado de 420 mil jóvenes (EL INFORMADOR, 2018), tal como pasa en la Universidad Nacional Autónoma de México (Unam), donde se acepta a menos del 10\% de los aspirantes.

Bajo este panorama, si el gobierno federal pretende dar una cobertura total a la Educación Superior necesita, por un lado, mejorar el presupuesto de las universidades y, por otro lado, proveer los recursos y medios tecnológicos y humanos necesarios para que crezcan tanto como sea necesario y puedan responder a la demanda actual de la población. 
c) Rescate de las universidades públicas autónomas de la crisis económica: En los últimos tres sexenios, las universidades públicas han sufrido recortes drásticos en el presupuesto asignado por la federación; dichos recortes han contribuido al colapso financiero de diez universidades del país (entre ellas, Universidad Autónoma de Chiapas, Universidad Autónoma del Estado de México, Universidad Michoacana de San Nicolás de Hidalgo, Universidad Autónoma de Morelos, Universidad Autónoma de Nayarit, Universidad Autónoma Benito Juárez de Oaxaca, Universidad Autónoma de Sinaloa, Universidad Juárez Autónoma de Tabasco, Universidad Veracruzana y Universidad Autónoma de Zacatecas). Ante dichas circunstancias, los programas educativos ofertados por estas universidades públicas están en riesgo, por lo que estas instituciones están imposibilitadas para abrir nuevos espacios.

d) Implementación de políticas para el uso eficaz del presupuesto: Las universidades también han sido un reflejo de la corrupción de los gobiernos anteriores; dentro de ellas se han construido cotos de poder en donde profesores y personal administrativo con amplio capital político gozan de pagos excesivos en las nóminas extraoficiales y no validadas en el subsidio ordinario de la SEP. Otro déficit tiene que ver con el pago de jubilados y pensionados con presupuesto que oficialmente debería destinarse a los trabajadores activos; asimismo, siguen existiendo contrataciones de personal docente que no cumplen con el perfil estipulado en la normatividad (MORENO, 2017). Ante dichas circunstancias, además de incrementar el financiamiento de las universidades, se necesita crear y aplicar políticas de transparencia para el uso eficaz del presupuesto asignado.

e) Tránsito hacia modelos educativos flexibles y pensar en sistemas educativos en línea: Las universidades necesitan transitar hacia modelos educativos flexibles que permitan una mayor cobertura de la población; para ello, los modelos de Educación en línea constituyen una alternativa relevante para el futuro (ALMEIDA et al., 2019). Cabe destacar que dicha estrategia no consiste en suplantar el modelo educativo presencial, sino de ampliar las oportunidades educativas para los jóvenes mexicanos. En el país existen algunos ejemplos de instituciones de Educación Superior que han implementado sistemas de Educación en línea de manera exitosa, como es el caso de la Universidad de Guadalajara (UDG) que en la última década ha diversificado su oferta educativa e incrementado su cobertura gracias a los programas educativos virtuales. Hoy en día, la UDG cuenta con más de 40 mil estudiantes inscritos en el sistema virtual (UDG, 2018), cifra que rebasa incluso la cantidad de alumnos inscritos en otras instituciones, como por ejemplo la Universidad de Colima que a finales de 2017 contaba con 27 mil 400 estudiantes matriculados (MORFÍN, 2017). 


\section{Educación en línea, una propuesta para el fortalecimiento de la cobertura en Educación Superior}

Como se ha visto en las últimas décadas, aunada a la rápida expansión de internet, la introducción de las Tecnologías de la Información y la Comunicación (TIC) en el ámbito educativo se ha multiplicado y justificado bajo el argumento de su potencial contribución a la mejora de los procesos educativos promovidos en las instituciones educativas formales (COLL, 2008). En un primer momento, esta mejora se vinculó a las posibilidades que dichas tecnologías tienen para promover experiencias innovadoras relacionadas con los métodos de enseñanza, los materiales educativos y las prácticas de evaluación (DÍAZ-BARRIGA, 2007, 2008) en los entornos formales presenciales; en un segundo momento, esta mejora se ha relacionado más con el desarrollo de entornos digitales de trabajo y aprendizaje que se presentan como espacios idóneos para mejorar tanto los procesos colaborativos como los resultados de aprendizaje de quienes participan en estos.

Sobre este segundo momento, la serie de informes Horizon emitidos desde el año 2012 a la fecha por parte de la New Media Consortium (NMC) han remarcado, año con año, un conjunto de tendencias y desafíos para las instituciones de Educación Superior que supone un cambio radical tanto en los espacios en los que comúnmente se despliegan los procesos de enseñanza y aprendizaje como en los propios roles o funciones del profesorado y de los alumnos que participan en estos nuevos espacios. Así, como el mismo NMC Horizon Report (EDUCAUSE, 2018) plantea, los grandes desafíos de hoy son aquellos que exigen avanzar en la "digital equity and adapting traditional organizational models to more flexible designs that advance the future of the workplace" (p. 6).

Como se ve, la incorporación de la tecnología en el ámbito educativo ya no es solamente un elemento de innovación o una herramienta de ayuda y mejora para los procesos de enseñanza y aprendizaje presenciales; de hecho, la tecnología y el acceso a internet se han vuelto un indicador de justicia social, ya que, de acuerdo con la propia Unesco (ECOSOC, 2011), la equidad digital es necesaria para promover la plena participación, comunicación y aprendizaje de las personas en este siglo.

La premisa anterior resalta un nuevo paradigma sobre la responsabilidad social de las instituciones educativas de nivel superior, ya que no se trata solamente de procurar el acceso a los recursos digitales, como materiales de aprendizaje 
de uso libre o repositorios, sino de aumentar el acceso a la Educación en línea y generar nuevas oportunidades educativas para aquellos que, difícilmente, podrían matricularse en las instituciones educativas tradicionales. Por lo que respecta a nivel nacional, este llamado de atención ha sido retomado y considerado por la SEP y la Anuies como uno de los principales ejes de gestión y proyectos en su Agenda 2015 para el desarrollo de la Educación Superior. En concreto, según los datos aportados en dicha agenda, México enfrenta una situación de atraso por el bajo nivel de cobertura de Educación Superior, ya que apenas alcanza el $34,1 \%$ frente a países que han superado el umbral del $50 \%$.

Sin duda, los datos nacionales sobre el nivel de cobertura, aunados a la nueva responsabilidad social que las instituciones de Educación Superior tienen hoy en día, señalan algunas implicaciones urgentes de atender en un nivel más macro. En efecto, las iniciativas gubernamentales deben encaminarse en, al menos, dos direcciones: primero, desarrollar estrategias que procuren la equidad digital de todos los mexicanos para garantizar su plena participación en los nuevos espacios de aprendizaje; y, segundo, utilizar u optimizar las tecnologías educativas con las que ya cuentan las instituciones educativas de nivel superior para aumentar el acceso a la Educación a través de distintas modalidades de Educación a distancia.

\section{Comentarios finales}

La mejora de la cobertura de Educación Superior y su universalización para garantizar las posibilidades de estudio a toda la población mexicana aparece ya como un planteamiento formal por parte del actual gobierno de México. Como el propio Ordorika Sacristán (2019) comenta, estas propuestas no solo recogen demandas históricas de los estudiantes universitarios que, mediante distintas luchas estudiantiles, han exigido el cumplimiento del derecho de acceso a la Educación Superior para todos los sectores, sino que además impone una serie de retos y desafíos que difícilmente pueden superarse en un solo sexenio, sobre todo cuando la propia situación de las instituciones de Educación Superior resalta la insuficiencia de la acción pública para atender los nuevos requerimientos de acceso, permanencia y logro educativo (MIRANDA LOPES; ISLAS DOSSETTI, 2016).

Como ya se ha comentado, no basta solamente con la construcción de nuevos recintos universitarios o la implementación de nuevos programas de becas económicas, mucho menos con la eliminación de los exámenes de admisión y la aplicación de políticas verticalistas; lo que realmente urge es tener un entendimiento profundo de las circunstancias por las que México sigue situándose -desde hace 
más de 15 años- como uno de los países con los niveles más bajos de cobertura de Educación Superior. En este sentido, como recomienda Solís (2018), resulta menester hacer un análisis longitudinal más detallado de la transición de la población por todos los niveles educativos, para identificar la naturaleza de los eventos que configuran la situación actual del sistema educativo mexicano.

Sin duda, la promesa de la cobertura total de Educación Superior no es fácil de cumplir, ya que como señalan algunos autores, no se trata de ofrecer a todos por igual lo que se ofrecía antes a unos pocos (MIRANDA LOPES, 2019; TENTI FANFANI, 2003); los esfuerzos que se encaminen en la mejora de la cobertura de Educación Superior deben poner el foco en, al menos, cinco ejes de discusión: 1) el respeto a la autonomía de las instituciones de nivel superior y la búsqueda del consenso de las políticas a implementarse con las universidades y los organismos relacionados con la promoción y el desarrollo de la Educación Superior en México; 2) la mejora del déficit en la infraestructura y capital humano en las universidades públicas otorgando los recursos financieros y medios tecnológicos y humanos necesarios; 3) el rescate de las universidades públicas autónomas que hoy en día enfrentan un fuerte colapso financiero; 4) la implementación de políticas para el uso eficaz y transparente del presupuesto asignado a las instituciones de Educación Superior; y 5) la promoción de modelos educativos flexibles y la puesta en marcha de sistemas educativos en línea.

Sobre este último punto, conviene destacar que pensar en los sistemas educativos en línea ya no es solamente una posible vía para alcanzar la tan deseada universalidad de la Educación Superior; de hecho, el acceso a la Educación en línea es ahora un indicador internacional de justicia social que exige desarrollar estrategias consensuadas en todos los niveles, para promover la equidad digital que garantice la participación y el aprendizaje de los mexicanos en este siglo. 


\section{Educação Superior no México: os desafios do governo presidencial no período 2018-2024 em matéria de cobertura}

\section{Resumo}

Uma das prioridades do atual Governo do México é elevar, para o ano de 2024, a taxa de cobertura em Educação Superior a 100\%. A referida meta implica em transformações importantes tanto na organização das universidades como no funcionamento e na operação dos programas educativos. Nesse artigo, apresenta-se uma análise sobre os desafios que o atual governo mexicano enfrenta em matéria de cobertura na Educação Superior e a pouca evolução que houve durante a última década; a partir dessa análise, propõem-se cinco eixos estratégicos que contribuem para a construção de politicas educacionais dirigidas ao fortalecimento da Educação Superior e à ampliação da matrícula dentro das universidades. Sugere-se que, para incrementar a taxa de cobertura, o desenvolvimento de políticas dirigidas ao fortalecimento e à diversificação de programas educativos online dentro das universidades.

Palavras-chave: Cobertura Educacional. Educação Superior. Universidades do México. Educação Online.

\section{Higher Education in Mexico: challenges of the current presidential government in the period 2018-2024 related to educational coverage}

\section{Abstract}

One of the priorities of the current government of Mexico expected for 2024 is to increase to $100 \%$ the educational coverage rate in Higher Education. Important transformations in the organization and operation of the universities as well as deeper changes on the operation of educational programs are necessary to achieve this goal. This article presents an analysis of the challenges facing the current Mexican government related to the little evolution of the educational coverage in Higher Education during the last decade. Based on this analysis, five strategic focuses are proposed to contribute to the development of educational policies aimed at strengthening Higher Education and expanding enrollment within universities. The development of policies aimed at strengthening and diversifying online educational programs within universities to increase the educational coverage rate are suggested.

Keywords: Educational Coverage. Higher Education. Online Education. Universities of Mexico. 


\section{Referencias}

ALMEIDA, A. N. et al. Effectiveness of public university expansion in Brazil: comparison between the situation of graduated and dropout students. Ensaio: Avaliação e Políticas Públicas em Educação, Rio de Janeiro, v. 28, n. 107, p. 457-479, abr.jun. 2020. https://doi.org/10.1590/s0104-40362019002701864

\section{ASOCIACIÓN NACIONAL DE UNIVERSIDADES E INSTITUCIONES DE EDUCACIÓN SUPERIOR - ANUIES. Anuarios estadísticos de educación superior. Ciudad de Mexico, 2018a. Disponible en: $\mathrm{http}$ //www.anuies.mx/informacion-y-servicios/informacion-estadistica-de- educacion-superior/anuario-estadistico-de-educacion-superior. Acceso en: 14 feb. 2019.}

ASOCIACIÓN NACIONAL DE UNIVERSIDADES E INSTITUCIONES DE EDUCACIÓN SUPERIOR - ANUIES. Visión y acción 2030: propuesta de la Anuies para renovar la educación superior en México: diseño y concertación de políticas públicas para impulsar el cambio institucional. Ciudad de Mexico, 2018b.

COLL, C. Aprender y enseñar con las TIC: expectativas, realidad y potencialidades. In: CARNEIRO, R.; TOSCANO, J. C.; DÍAZ, T. (comps.). Los desafios de las TIC para el cambio educativo. Madrid: Fundación Santillana, 2008. p. 113-126.

DÍAZ BARRIGA, F. Educación y nuevas tecnologías de la información: ¿hacia un paradigma educativo innovador? Sinéctica, Revista Electrónica de Educación, Tlaquepaque, n. 30, p. 1-15, ene./jun. 2008.

DÍAZ BARRIGA, F. La innovación en la enseñanza soportada en TIC: una mirada al futuro desde las condiciones actuales. In: SEMANA MONOGRÁFICA DE EDUCACIÓN, 22., Madrid, 2007. Actas[...] Madrid: Fundación Santillana, 2007. Disponible en: http://www.oei.es/tic/santillana/ Barriga.pdf. Acceso en: 10 feb. 2019.

EDUCAUSE. NMC Horizon report preview: 2018 higher education edition. Louisville, 2018.

EL INFORMADOR. Universidades rechazan a 420 mil al año. Noticias Mexico, 2 ago. 2018. Disponible en: https://www.informador.mx/ Universidades-rechazan-a-420-mil-al-ano-1201808020003.html. Acceso en: 2 mar. 2019. 
GÓMEZ, P. El examen de admisión sí es una mentira. Proceso Opinion, 30 mar. 2018. Disponible en: https://www.proceso.com.mx/527912/elexamen-de-admision-si-es-una-mentira. Acceso en: 16 feb. 2019.

GONZÁLEZ, H. Cobertura en educación superior: de los compromisos a la realidad. Nexos, 25 nov. 2015. Disponible en: https://educacion.nexos.com. $\mathrm{mx} / \mathrm{p}=94$. Acceso en: 16 feb. 2019.

GONZÁLEZ, H. ¿Qué hacemos con los rechazados de las universidades?

Nexos, 5 sept. 2018. Disponible en: https://educacion.nexos.com.mx/?p=1500. Acceso en: 25 feb. 2019.

INSTITUTO NACIONAL PARA LA EVALUACIÓN DE LA EDUCACIÓN - INEE. Panorama educativo de México: indicadores del sistema educativo nacional 2016. Ciudad de Mexico, 2016.

MARCHESI, A.; BLANCO, R.; HERNÁNDEZ, L. (coords.). Avances y desafios de la educación inclusiva en Iberoamérica. Madrid: Organización de Estados Iberoamericanos para la Educación, la Ciencia y la Cultura, 2014. Disponible en: http://www.oei.es/publicaciones/Metas inclusiva.pdf. Acceso en: 28 ene. 2019.

MÁRQUEZ JIMÉNEZ, A. A 15 años de PISA: resultados y polémicas. Perfiles Educativos, Ciudad de Mexico, v. 39, n. 156, p. 3-15, abr./jun. 2017. https://doi.org/10.22201/iisue.24486167e.2017.156.58280

MEXICO. Ministério de Educación, Cultura y Deportes. Principales cifras del sistema educativo nacional 2016-2017. Ciudad de Mexico, 2017.

MENDOZA ROJAS, J. Situación y retos de la cobertura del sistema educativo nacional. Perfiles Educativos, Ciudad de Mexico, v. 40, n. esp. 156, p. 119-140, nov. 2018. https://doi.org/10.22201/iisue.24486167e.2018.Especial.59179

MIRANDA LOPES, F. Abandono escolar en educación media superior: conocimiento y aportaciones de política pública.

Sinéctica, Tlaquepaque, n. 51, p. 1-22, jul./dic. 2018. https://doi.org/10.31391/S2007-7033(2018)0051-010

MIRANDA LOPES, F. Diagnóstico, teoría e intervenciones públicas para abatir el abandono escolar en la educación secundaria de segundo ciclo: aprendizajes desde América Latina. Revista Latinoamericana de Educación Comparada, Buenos Aires, v. 9, n. 14, p. 11-30, nov./mayo 2019. 
MIRANDA LOPES, F.; ISLAS DOSSETTI, J. Los jóvenes, sus derechos y la equidad en la educación media superior en México: hacia una agenda de política educativa. Polifonías Revista de Educación, Buenos Aires, v. 5, n. 9, p. 149-177, sept./oct. 2016.

MORENO-BRID, J. C.; RUIZ-NÁPOLES, P. La educación superior y el desarrollo económico en América Latina. Revista Iberoamericana de Educación Superior, Ciudad de Mexico, v. 1, n. 1, p. 171-188, ene. 2010.

MORENO, T. SEP: no se rescatará a universidades quebradas. El Universal, 21 nov. 2017. Disponible en: https://www.eluniversal.com.mx/nacion/politica/ sep-no-se-rescatara-escuelas-quebradas. Acceso en: 12 dic. 2018.

MORFÍN, U. U de Colima incremente 5\% matrícula, hay 27 mil 400 alumnos: rector. AF Medios, 13 dic. 2017. Disponible en: https://www.afmedios. com/2017/12/universidad-de-colima-incrementa-matricula-27-mil-400alumnos-rector/. Acceso en: 12 dic. 2018.

ORDORIKA SACRISTÁN, I. Educación superior pública, autónoma, gratuita y obligatoria. In: FORO IMPLICACIONES DE LA OBLIGATORIEDAD Y GRATUIDAD DE LA EDUCACIÓN SUPERIOR EN MÉXICO., 2019, Ciudad de Mexico. Actas[...]. Ciudad de Mexico: Asociación Nacional de Universidades e Instituciones de Educación Superior, 2019. Disponible en: https://ses.unam.mx/ integrantes/uploadfile/iordorika/Ordorika_Audiencia.pdf. Acceso en: 22 feb. 2019.

\section{ORGANISATION FOR ECONOMIC CO-OPERATION AND} DEVELOPMENT - OECD. Panorama de la educación 2017: indicadores de la OCDE. Madrid: Fundación Santillana, 2017.

OROZCO CRUZ, J.; OLAYA TORO, A.; VILLATE DUARTE, V. ¿Calidad de la educación o educación de calidad? Una preocupación más allá del mercado. Revista Iberoamericana de Educación, Madrid, v. 51, p. 161-181, 2009. https://doi.org/10.35362/rie510637

RAMOS SERPA, G.; LOPEZ FALCÓN, A. Masificación, equidad, educación superior: la universalización de la educación superior cubana. Ensaio: Avaliação e Políticas Públicas em Educação, Rio de Janeiro, v. 27, n. 103, p. 291-316, abr./jun. 2019. https://doi.org/10.1590/s0104-40362019002701783

SÁNCHEZ JIMÉNEZ, A. Reconoce Anuies rezago en cobertura educativa a nivel superior. La Jornada Sociedad y Justicia, 19 ago. 2018. Disponible en: https://www.jornada.com.mx/2018/08/19/sociedad/029n2soc\#. Acceso en: 20 dic. 2018. 
SOLÍS, P. La transición de la secundaria a la educación media superior en México: el difícil camino a la cobertura universal. Perfiles Educativos, Ciudad de Mexico, v. 40, n. 159, p. 66-89, ene./mar. 2018. https://doi.org/10.22201/iisue.24486167e.2018.159.58412

TENTI FANFANI, E. (ed.). Educación media para todos: los desafíos de la democratización del acceso. New York: United Nations Children's Emergency Fund, 2003.

UNITED NATIONS CHILDREN'S EMERGENCY FUND - UNICEF. El derecho a la educación: una tarea pendiente para América Latina y el Caribe. Desafios 3, ago. 2006. Disponible en: https://repositorio. cepal.org/bitstream/handle/11362/35991/Desafios_Nro3_esp_ es.pdf? sequence=1\&isAllowed=y. Acceso en: 4 mar. 2019.

UNITED NATIONS ECONOMIC AND SOCIAL COUNCIL - ECOSOC. Educación de calidad en la era digital: una oportunidad de cooperación para Unesco en América Latina y el Caribe. Santiago: Oficina Regional de Educación para América Latina y el Caribe, 2011. Disponible en: http://www.unesco.org/new/fileadmin/MULTIMEDIA/FIELD/Santiago/pdf/ educacion-digital-Buenos-Aires.pdf. Acceso en: 23 ene. 2019.

\section{UNITED NATIONS EDUCATIONAL, SCIENTIFIC AND} CULTURAL ORGANIZATION - UNESCO. Directrices sobre políticas de inclusión en la educación. New York, 2009. Disponible en: http://unesdoc.unesco.org/images/0017/001778/177849s.pdf. Acceso en: 23 ene. 2019.

UNIVERSIDAD DE GUADALAJARA - UDG. Estadísticas de alumnos. Ciudad de Mexico, 2018. Disponible en: http://www.escolar.udg.mx/ estadisticas/alumnos/2018. Acceso en: 3 mar. 2019. 


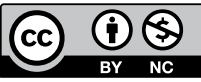

\section{Información de los autores}

Juan Carlos Castellanos-Ramírez: Doctor en Psicología de la Educación por la Universidad de Barcelona, España. Profesor Investigador de Tiempo Completo en la Universidad Autónoma de Baja California, México. Miembro del Sistema Nacional de Investigadores, México. Contacto: juan.castellanos8@uabc.edu.mx

iD https://orcid.org/0000-0002-0682-9085

Shamaly Alhelí Niño Carrasco: Doctora en Psicología de la Educación por la Universidad de Barcelona, España. Profesora Investigadora de Tiempo Completo en la Universidad Autónoma de Baja California, México. Miembro del Sistema Nacional de Investigadores, México. Contacto: shamaly.nino@uabc.edu.mx

iD https://orcid.org/0000-0002-5623-4418 